\title{
Clinico-epidemiological profile of confirmed cases of dengue infection: A tertiary care teaching hospital based study of central India
}

\author{
Deepti Gupta ${ }^{1}$, Veena Maheshwari ${ }^{2, *}$, Deepankar Parmar ${ }^{3}$ \\ ${ }^{1,2}$ Assistant Professor, ${ }^{3}$ Professor, ${ }^{1,3}$ Dept. of Pathology, ${ }^{2}$ Dept. of Microbiology, RKDF Medical College Hospital \& Research \\ Centre, Bhopal, Madhya Pradesh, India \\ *Corresponding Author: Veena Maheshwari \\ Email: mail2veenam@yahoo.co.in
}

Received: $11^{\text {th }}$ May, 2018

Accepted: $14^{\text {th }}$ June, 2018

\begin{abstract}
Introduction: Dengue is a common vector borne ailment caused by dengue virus \& transmitted by aedes mosquitoes. It is a significant cause of morbidity in India presenting with wide range of severity \& leading to complications like DHF and DSS. The present study was done to assess the clinico-epidemiological features of dengue cases attending the tertiary care hospital of Gwalior, Madhya Pradesh.

Materials and Methods: A prospective analytical study was carried out in the Pathology Department, GRMC Gwalior, Madhya Pradesh, for a period of one year (Sept 2011- Oct 2012). Total 120 clinically suspected patients of dengue fever were evaluated by serological analysis for confirmation of cases.

Results. Out of the 48 confirmed dengue cases majority of the cases i.e. 89.25\%.were in the age group of 16-45 years. Males outnumbered the females giving M: F ratio of 2.2:1. All confirmed cases reported with fever. Around (85.4\%) and (79.2\%) had complaint of headache and bodyache.

Conclusion: Dengue is an important public health problem affecting predominantly the male urban population of age group of 16-45yrs. Classical cases have clinical symptoms of fever, headache and bodyache which vary with virus strain, age and immune status of the host. Early diagnosis based on clinical profile, laboratory investigations along with proper management, community awareness, vector control measures and lastly constant vigilance by the health-care officials can change the situation in combating dengue infection.
\end{abstract}

Keywords: Dengue, Clinico-epidemiological, Central India.

\section{Introduction}

Dengue is one of the most frequent and widespread arboviral infections in humans, transmitted primarily by Aedes aegypti mosquitoes. In the last 5 decades, there has been a remarkable global increase in number of dengue cases affecting all age groups. It has become a serious public health problem, specifically in the tropical and sub-tropical countries because of lack of antiviral drugs or vaccine. Around 50 million dengue infections arise annually and approximately 2.5 billion people live in dengue endemic countries. ${ }^{1}$ World Health Organization (WHO) has conferred it as a notifiable disease and since 2005 dengue is considered as a public health emergency of international concern. ${ }^{2-4}$

Dengue was reported first in 1780, when Benjamin Rush explained this condition as "break bone fever". 5 Dengue virus (DV) is classified under family Flaviviridae. Four serotypes of this virus have been detected referred to as DV-1, DV-2, DV-3, and DV-4, which have been isolated in India. ${ }^{6}$

Most dengue virus infections are subclinical. The Dengue virus infection shows a wide range of severity that may be classical dengue fever (DF) to the complicated dengue hemorrhagic fever (DHF) and Dengue shock syndrome (DSS), the unusual manifestations including encephalitis, liver failure and severe bleeding without shock.
Classical dengue fever (DF) presents clinically after an incubation period of 3-14 days as fever of sudden onset with headache, retro orbital pain, conjunctival injection, musculoskeletal pain, pain in the back and limbs (Break bone fever), lymphadenopathy and maculopapular rash. ${ }^{7}$ The fever is typically biphasic (saddle back) and lasts for 5-7 days. Other common symptoms include weakness, abdominal pain, sore throat and headace and vomiting, but the severity of illness and clinical manifestations vary with age; virus strain and immune status of the host.

Major demographic changes such as uncontrolled population growth, unplanned urbanization resulting in substandard housing and need for water storage has greatly facilitated vector proliferation. ${ }^{8}$ The infection by the dengue virus of one serotype provides lifelong immunity to that serotype, but gives only partial and transient protection against subsequent infection by the other serotypes and sequential infection with different serotypes increase the risk of developing DHF. ${ }^{5}$ There is currently no specific treatment is available and potential vaccines are also in the developmental stage, therefore, the only method of protecting against DENV transmission is vector control.

Proper diagnosis is the most challenging aspect of dengue infection. The characteristic laboratory finding for the disease is leucopenia and other laboratory 
findings include thrombocytopenia, elevations of serum aminotransferases. ${ }^{9}$

The gold standard test (viral isolation by cell culture) for identification of Dengue virus infection is not approachable of peripheral and even most tertiary care laboratories and the diagnosis is mainly based on detection of Dengue specific IgM/IgG antibodies. ${ }^{10}$

Dengue infection presents as a very nonspecific illness and is hardly recognized as a clinical entity by primary health care physicians. So its diagnosis still is a challenge for the treating physician. Early detection and initiation of appropriate treatment may alter the course of infection and improve the prognosis.

Despite being lot of cases reported in Gwalior every year, much data is not available on the clinicoepidemiological profile of DF cases, so present study was done to studying the clinico-epidemiological features of dengue cases attending a tertiary care teaching hospital of central India.

\section{Materials and Methods}

In association with Defense Research and Development organization (Ministry of Defense, Government of India), this cross-sectional prospective study was carried out in the Department of Pathology of Gajra Raja Medical College, Gwalior, Madhya Pradesh, India, from 1st Sept. 2011 to 31st Oct. 2012. All ethical clearances were obtained from Medical College Research Ethical Committees. Patients of either sex and of any age showing signs and symptoms suggestive of dengue fever were included in the study. A written consent was taken after informing to the patient, patient's parents (in case of minor) or patient's relatives before conducting this study. A total of 120 serum samples were collected from clinically suspected dengue cases attending OPD or admitted in the wards of J.A. Group of Hospitals, Gajra Raja Medical College, Gwalior, Madhya Pradesh.

A clinically suspected case of dengue which was included in the study was considered, a patient with an acute febrile illness with $\geq 2$ of the following manifestations: headache, retro-orbital pain, myalgia, arthralgia, rash, and hemorrhagic manifestation as per WHO criteria. Those patients were excluded from the study who had concomitant malaria, typhoid or any other infection.

A pre formed data sheet was used as a tool for data collection. The collected data was subjected to statistical analysis using Microsoft excel.

\section{Result}

All suspected cases of dengue fever were tested for dengue serology by the rapid card test (NS1 Ag and IgG and IgM $A b)$.

During the study period, a total of 120 (M=86 and $\mathrm{F}=34$ ) patients were suspected to have dengue infection. Out of these total 120 patients, only 48 cases were found to be positive for the Dengue virus infection [Table 1]

Clinically there was no case identified for dengue hemorrhagic fever among the total 120 patients.

Out of the 48 confirmed dengue cases, a total of 34 (70.83\%) patients were from urban area and $14(29.2 \%)$ from rural area [Table 2]

Fig. 2 shows the distribution of dengue positive cases in various age groups in either sex. Out of the 48 serologically positive cases, $33(27.5 \%)$ cases were males and $15(12.5 \%)$ cases were females. Among the males, 15 cases were from the 16-30 years age group, 9 cases were from the 31-45 years age group, 4 cases were from the $<15$ years age group, 2 cases were from the 46-60 years age group and 1 case was from the $>60$ years age group.

Among the females, 10 cases were from the 16-30 years age group, 5 cases were from the 31-45 years age group, 2 cases were from the $<15$ years age group. [Fig. 2]

Male to female ratio was reported to be $2.2: 1$. The majority of cases belonged to the age group of 16-30 yrs and males clearly outnumbered females.

In the present study, the most common clinical presentation was fever that was found among all the $48(100 \%)$ patients. The fever was of a mild to moderate grade in most of the patients and showed no specific pattern. Fever was followed by Headache in $41(85.4 \%)$ cases. Bodyache was noted in $38(79.2 \%)$ cases, Nausea \& Vomiting in $27(56.2 \%$ ) cases, retro-orbital pain in 14 (29.2) cases, abdominal pain in $13(27 \%)$ cases, joint pain in $12(25 \%)$ cases, myalgia in $11(22.9 \%)$ cases and rash in $8(16.7 \%)$ cases. (Table 3$)$

Table 1: Seroprevalance of dengue cases

\begin{tabular}{|c|c|c|}
\hline $\begin{array}{c}\text { Total no. of } \\
\text { patients }\end{array}$ & $\begin{array}{c}\text { Dengue positive } \\
\text { patients }\end{array}$ & $\%$ \\
\hline 120 & 48 & $40 \%$ \\
\hline
\end{tabular}

Table 2: Geographical distribution of dengue cases

\begin{tabular}{|l|c|c|}
\hline \multicolumn{1}{|c|}{ Area } & $\begin{array}{c}\text { Number of } \\
\text { patients }\end{array}$ & $\boldsymbol{\%}$ \\
\hline Urban & 34 & $70.80 \%$ \\
\hline Rural & 14 & $29.20 \%$ \\
\hline Total & 48 & $100 \%$ \\
\hline
\end{tabular}

Table 3: Clinical manifestations of dengue cases

\begin{tabular}{|c|c|c|}
\hline $\begin{array}{c}\text { Clinical } \\
\text { manifestation }\end{array}$ & $\begin{array}{c}\text { No. of } \\
\text { Patients }\end{array}$ & $\mathbf{\%}$ \\
\hline Fever & 48 & $100 \%$ \\
\hline Headache & 41 & $85 \%$ \\
\hline Bodyache & 38 & $79.20 \%$ \\
\hline Nausea-Vomiting & 27 & $56.20 \%$ \\
\hline Retro-orbital pain & 14 & $34.20 \%$ \\
\hline Joint Pain & 12 & $25 \%$ \\
\hline Myalgia & 11 & $25.80 \%$ \\
\hline Abdominal pain & 13 & $17.50 \%$ \\
\hline Rashes & 8 & $10 \%$ \\
\hline
\end{tabular}




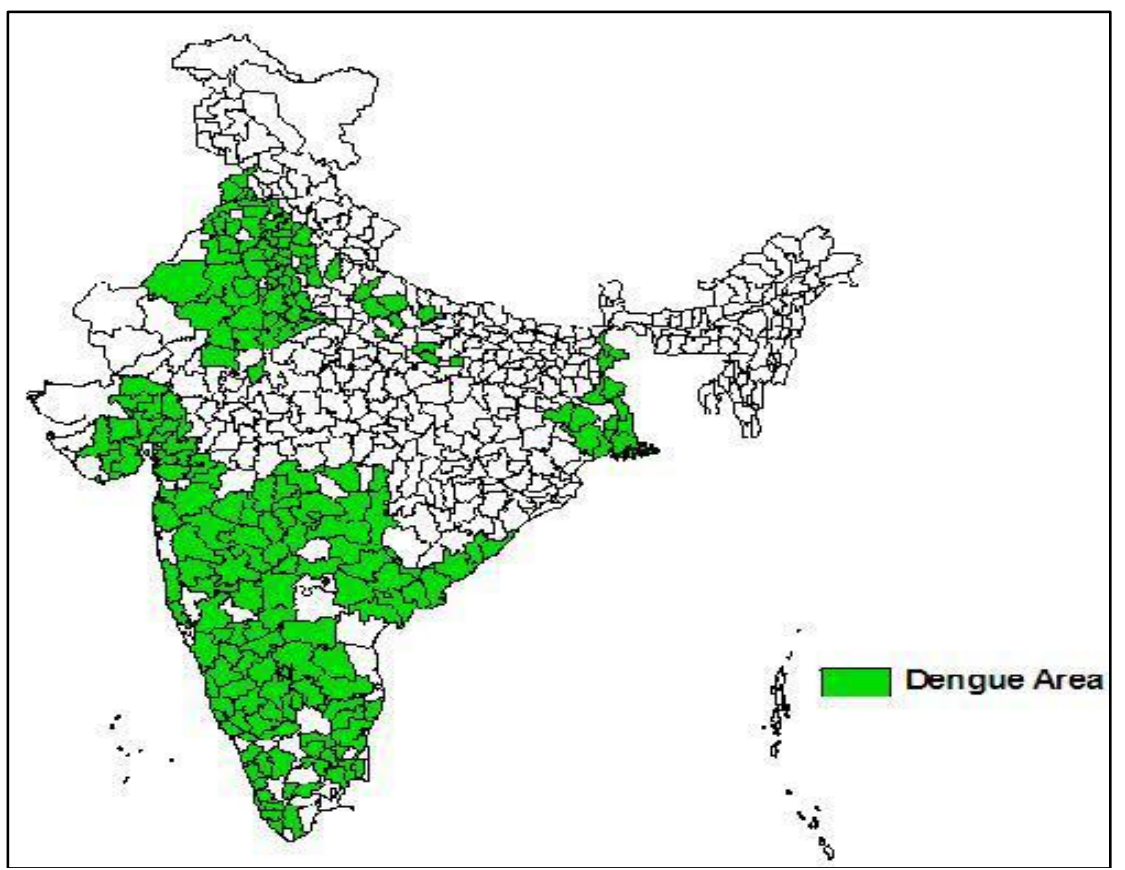

Fig. 1: Distribution of dengue in various regions of India

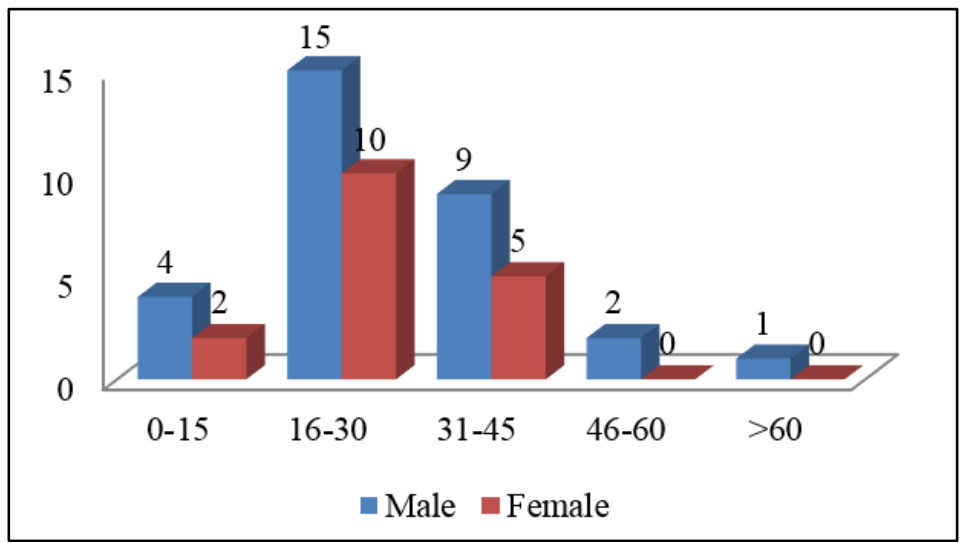

Fig. 2: Age and Sex distribution of total dengue confirmed cases

\section{Discussion}

Dengue fever continues to be a major public health problem in India affecting all age groups and both sexes. Dengue today ranks as the most important vector borne ailment, with about 2.5 billion people in two hundred countries at risk.

Dengue infections vary in severity, ranging from influenza-like self-limiting illness to life threatening DHF and DSS which, if left untreated, is associated with significant mortality as high as $20 \% .^{11}$ The resurgence of dengue has been noted in India and dengue outbreaks have been frequently reported in the country from different parts in both urban and rural populations. $^{12}$

In the present study, $89.25 \%$ cases belonged to age group of 16-45 years. It could be explained on the basis of the fact that the individuals in this age group are economically active; they usually get involved in outdoor as well as household activities due to which there are higher chances of exposure to vectors and therefore contact infection. These findings were comparable with a study conducted by Saini et $\mathrm{al}^{13}$ in Western Maharashtra, India. A similar study done in Delhi, North India conducted by Ahmed $\mathrm{N}$ et al reported $70 \%$ cases in the age group 11-40 years. ${ }^{14}$

These observations were also consistent with a study done by Kendre VV et al in 2014 who found that most cases occur in 15-44 yrs. of age. ${ }^{15}$

In our study, $33(68.75 \%)$ patients were males and $15(31.25 \%)$ were females with a male to female ratio of 2.2:1. Higher incidence of dengue infection in males could be explained on the basis of that, males predominantly form the working population and are more prone to acquire mosquito borne dengue infections in day time. Ashwini Kumar et $\mathrm{al}^{16}$ in Karnataka and Karoli et al. ${ }^{17}$ in North India also showed the male preponderance in their studies. 
Arti Jain $(2017)^{10}$ in the study done in central India, noted male female ratio of 2:1. Ashwini Kumar et al $(2010)^{16}$ in his study in udupi (Karnataka) found male female ratio as 1.8:1. Our results were consistent with their findings.

In this study, a total of $34(70.83 \%)$ patients were from urban area where as $14(29.2 \%)$ cases were from rural area. As our hospital is associated with medical college in city area, a majority of patients were from urban area.

Seasonal prevalence of dengue during the study period 2011-12, revealed that majority of cases were detected during post monsoon season i.e. SeptemberNovember. Virus activity is high during monsoon and post monsoon period which coincides with increased vector breeding

The patients with dengue fever classically present with the fever of sudden onset, weakness, frontal headache, retro-orbital pain, and backache along with severe myalgia. ${ }^{18}$ In our study the clinical profile of dengue patients revealed that fever was the most common clinically presenting symptom present in all $48(100 \%)$ cases followed by Headache $41(85.4 \%)$ and Bodyache 38 (79.2\%). Similar studies in and around India ${ }^{9,16,19-21}$ have also substantiated fever as being the most common presenting symptom. The other clinical features noted in our study were headache, nausea vomiting, abdominal pain, rashes, myalgia, retro-orbital pain, as reported in previous studies. . $2,23^{2}$

Out of total 120 cases, 48 cases found to be positive for dengue by serology showing the $40 \%$ seroprevalence of dengue in this study. Madan et al $(2018)^{24}$ in their study done in Maharashtra found $21.6 \%$ seroprevalence and $31.3 \%$ seroprevalence was noted by Ukey et al $(2010)^{25}$ in their study. In an study done by P. Jyoti and B Metri (2015) $18.99 \%$ seroprevalence of dengue was reported. The variation noted in different studies may be due to reasons that presentation of dengue is very non specific initially so diagnosis is not easy and also geographical and entomological factors.

\section{Conclusion}

To conclude, Dengue is a notifiable disease and also an important international public health problem which is prevalent in tropical and sub-tropical countries affecting predominantly the male urban population of age group of 16-45 yrs. Classical cases have clinical symptoms of fever, headache and Bodyache which vary with virus strain, age and immune status of the host. Early diagnosis based on clinical profile, laboratory investigations along with proper management, community awareness, vector control measures and lastly constant vigilance by the health-care officials can change the situation in combating dengue infection.

\section{References}

1. World Health Organization, Dengue: guidelines for diagnosis, treatment, prevention and control-new edition. A joint publication of the World Health Organization (WHO) and the special program for research and training in tropical diseases (TDR) 2009.

2. Kale AV. clinical profile and outcome of dengue fever from a tertiary care centre at Aurangabad Maharashtra India: an observational study, IOSR journal of dental and medical sciences. 2014;13(9):14-19.

3. Chatterjee $\mathrm{N}$ et al, an observational study of dengue fever in a tertiary care hospital of eastern India, journal of the association of physicians of India. 2014;62:224-227.

4. S. Ahammad F. Clinico-demographic profile of dengue fever in a southindian tertiary care teaching hospital. World Journal of Pharmacy and Pharmaceutical Sciences. 2016;5(1):1602-1609.

5. Makwana A, Shrivastava S, Mehta M, Chatterjee S. Clinical profile of dengue fever in tertiary care hospital, Jamnagar Gujrat. IJAR. 2016:2(3):636-638.

6. Oza JR, Kanabar BR, Patel UV, Gajera, KD, Thakrar DV, Jogia AD. Clinico-Haematological Profile and Outcome of Dengue Fever Cases Admitted In s2014 at Tertiary Care Hospital, Rajkot, Gujarat. Natl J Community Med. 2017;8(6):320-323.

7. Anantnarayan R, Paniker C.: Textbook of Microbiology $9^{\text {th }}$ Ed. Universities press, Hyderabad:2013;288-301.

8. Naik JD, Jain SR, Babar S. Clinico-epidemiological study of dengue cases admitted in tertiary care hospital: record based study. J Evolution Med Dent Sci. 2016;5(29):14881491. DOI: $10.14260 /$ jemds/2016/350

9. Shah V, Jain U. Clinical profile of patient with dengue fever in a tertiary care teaching hospital. Int J Med Sci Public Health. 2017;6:165-168.

10. Arti Jain. 2017. Occurrence of Dengue Fever in Tertiary Care Hospitals. Int J Curr Microbiol App Sci. 6(2):10851089.

11. WHO: Dengue Guidelines for Diagnosis, Treatment, Prevention and Control. Available at: http://whqlibdoc.who.int/publications/2009/97892415478 71_eng.pdf. Accessed July 5th, 2012.

12. Gupta P, Khare V, Tripathi S. Assessment of WHO definition of dengue hemorrhagic fever in North India. $J$ Infect Dev Ctries. 2010;4:150-155.

13. S. Saini, Anagha G Kinikar: Epidemiology and seropositivity of dengue fever cases in a rural tertiary care hospital of western Maharashtra, India. International Journal of Biomedical and Advance Research. 2013;4(7):743-749.

14. Ahmed N! H, Broor S. Dengue Fever outbreak in Delhi,North India: A Clinico- Epidemiological study. Indian J Community Med. 2015;40:135-8.

15. Kendre VV, Shekde SD, Chinte LT. The study of clinicoepidemiological features of dengue cases admitted in tertiary care hospital, latur, Maharashtra. International Journal of Recent Trends in Science and Technology. 2014;10(2):369-372.

16. Ashwini Kumar, Chythra R Rao, Vinay Pandit, et al. Clinical Manifestations and Trend of Dengue Cases Admitted in a Tertiary Care Hospital, Udupi District, Karnataka. Indian J Community Med. 2010;35(3):386390.

17. Ritu Karoli, Jalees Fatima, Zeba Siddiqi et.al. Clinical profile of dengue infection at a teaching hospital in North India. J Infect Dev Ctries. 2012;6(7):551-554. 
18. Kuhn JH, Peters CJ. Arthropod-borne and rodent-borne virus infections. In: Kasper DL, Fauci AS, Hauser SL, Longo DL, Jameson JL, Loscalzo J, editors. Harrison's Principles of Internal Medicine. 19th ed. Volume 2. McGraw-Hill Education; Columbus, OH, USA: 2015:1304-23.

19. Kabilan L, Balasubramanian S, Keshava SM, Satyanarayana K. The 2001 Dengue epidemic in Chennai. Indian J Paediatr. 2005;72:919-23.

20. Kumar R, Tripathi P, Tripathi S, Kanodia A, Pant S, Venkatesh $\mathrm{V}$. The prevalence and the clinical differentiation of Dengue fever in children in northern India. Infection. 2008;36:444-49.

21. Kumar H, Mansoor SB. A study of clinico-demographic profile of dengue cases in a teaching hospital. National Journal of Laboratory Medicine. 2015;4(3):22-26.

22. Ratageri VH, Shepur TA, Wari PK, Chavan SC, Mujahid IB, Yergolkar PN. Clinical profile and outcome of Dengue fever cases. Indian J Pediatr. 2005;72(8):705-6.
23. Shahin W, Nassar A, Kalkattawi M, Bokhari H. Dengue fever in a tertiary hospital in Makah, Saudi Arabia. Dengue Bull. 2009;33:34-44.

24. Madan SP, Bhatawadekar S, Lahiri K. clinicodemographic profile and seroprevalence of dengue at a tertiary care hospital- study from Maharashtra. Int $J$ Health Sci Res. 2018;8(1):43-48.

25. Ukey PM, Bondade SA, Paunipagar PV, Powar RM, Akulwar SL. Study of seroprevalence of Dengue fever in Central India. Ind J Community Med. 2010;35(4):517-19.

26. Parameswarappa Jyothi, Basavraj C Metri. Correlation of serological markers and platelet count in the diagnosis of Dengue virus infection. Adv Biomed Res. 2015;4:26.

How to cite this article: Gupta D, Maheshwari $\mathrm{V}$, Parmar D. Clinico-epidemiological profile of confirmed cases of dengue infection: A tertiary care teaching hospital based study of central India. Indian J Pathol Oncol. 2018;5(4):531-535. 